\title{
SUPPLIER-INDUCED DEMAND FOR PHYSIOTHERAPY IN THE NETHERLANDS
}

\author{
E. VAN DOORsLaer and J. Geurts \\ Department of Health Economics, University of Limburg, P.O. Box 616, 6200 MD Maastricht, \\ The Netherlands
}

\begin{abstract}
Empirical studies of supplier-induced demand in health care have mostly concentrated on the analysis of physician behaviour. In this article, the focus is on the economic determinants of physiotherapist behaviour in The Netherlands. It is shown that relative prices work as strong incentives to alter the mix of services supplied, conform to the model of revenue maximization under a production constraint. However, the time-series analysis also gives some indication that this ability to influence the demand for their services to increase hourly income is not fully exploited. The latter finding is inconsistent with pure income maximization but rather points to a trade-off between loss of revenue and demand manipulation. The fact that the choice of therapy varies with the pressure on provider incomes does cast some doubt on the appropriateness of the chosen patterns of treatment in terms of effectiveness.
\end{abstract}

Key words - supplier-induced demand, physiotherapy, economic incentives

\section{INTRODUCTION}

Health economists studying provider behaviour have generally been concerned with the behaviour of physicians as the most important decision makers in health care. A large number of behavioural models have been developed to explain such peculiar characteristics as supplier-induced demand (for reviews, see e.g. $[1,2]$ ). Less effort has been made to model the economic behaviour of paramedical personnel. This may partly be due to the fact that they, very often, are not responsible for important resource allocation decisions. This article illustrates that this neglect is not always justified.

We have studied the behaviour of self-employed physiotherapists in The Netherlands. A model of rational revenue-maximizing behaviour is developed which takes into account the peculiarities of this market. We also propose an empirical test of supplier-induced demand which does not look at the volume but rather at the mix of services provided by physiotherapists. The predictions are tested by means of a time series analysis of physiotherapy utilization rates. In the period considered, new government regulatory measures were introduced with regard to both the volume and prices of physiotherapeutic care delivered to publicly insured patients. The variation caused by these interventions enabled us to test for a response in provider behaviour. The major question we seek to answer is whether, how and to what extent the choice of therapy is determined by economic variables such as prices and incomes. The results are discussed in view of the agency role of the physiotherapist for patient and society.

\section{DEMAND FOR AND SUPPLY OF PHYSIOTHERAPY IN THE NETHERLANDS}

In The Netherlands physiotherapeutic care is completely covered under public health insurance and subject to a number of regulations [3]. Publicly insured patients $(70 \%$ of the Dutch population) cannot consult a physiotherapist on their own initiative but have to be referred by their GP or specialist. Patients only express an undifferentiated demand for care by consulting a physician. The referring physician prescribes the number of physiotherapeutic treatments (visits) to be given but in general leaves the decision about the composition of the treatment to the physiotherapist. A typical 'combined treatment' consists of massage/exercise therapy possibly in combination with one or more so-called 'applications' which are forms of therapy that make use of electricity, heat or ultrasound. Treatment is completely free of charge to the public patient. The physiotherapist working in private practice is remunerated on the basis of a fee per item of service. Because the fees differ according to the type of therapy, it is clear that this situation creates incentives for the providers to manipulate the demand for their services. A considerable amount of uncertainty about the effectiveness of most forms of therapy, and therefore the lack of firm indications as to which therapy is appropriate for which diagnosis, increases the freedom of choice of therapy for the physiotherapist. It is not surprising then to find that a large variation in treatment patterns across practices remains even after correction for diagnosis. Whether this variation is merely random and thus a consequence of professional uncertainty [4] or has also got something to do with rational economic behaviour is a question in which we are interested here.

Complete coverage of expenditures for publicly insured patients on the demand side, and an unrestricted rapid growth of the number of physiotherapists remunerated fee-for-service on the supply side, have caused an increase of physiotherapeutic expenditures in The Netherlands, which is much higher than the general increase in health care expenditures. While undeflated total health care costs have tripled between 1972 and 1982, the costs for physiotherapeutic care were in 1982 five-fold the level 
of 1972. Troubled by this rapid growth, the government introduced some new regulation concerning tariffs and volume of physiotherapeutic care provided to publicly insured patients.

The fee measure was introduced 1 October, 1979. Before that time, there were only two fees: one for massage/exercise therapy and one for the technical applications. However, because the average duration of each application is different, the marginal revenue for the physiotherapist per unit of time spent using an application differed also. To the degree that substitution is possible and the physiotherapist's time input scarce, applications with a shorter average duration were clearly more attractive. Substitution was, however, not strictly necessary since it was also allowed to perform more than one application per treatment. With the introduction of the fee measure "standard durations in minutes' were attached to all forms of therapy but the fee per minute was the same for every activity. Of course, this implied some drastic changes in the fees per type of therapy. As can be seen from Table 1, all fees were lowered except for massage/exercise and low-frequency electrotherapy.

The second measure, here after called volume measure, was implemented on 1 April, 1980 and was aimed at limiting the use of technical applications because of their doubtful effectiveness. Series of more than 12 combined treatments (massage plus application) were no longer allowed, nor was the use of more than one application per treatment.

\section{AN ECONOMIC MODEL OF PHYSIOTHERAPIST BEHAVTOUR}

In this section we will illustrate the behaviour of Dutch physiotherapists by means of the optimization problem faced by a firm which is jointly producing multiple outputs. The model could, however, be applied to any health care provider, who can choose between different compositions of treatment mix under certain constraints [5].

Consider a physiotherapist who has to produce a given number of visits $V^{0}$, which is the input that is exogenously determined by the referring physicians. Given his initial endownments of labour and capital (which are assumed to be fixed in the short run) a production possibility frontier exists which represents all combinations of two applications $A_{1}$ and $A_{2}$ that can be produced in $V^{0}$ visits. Such a transformation curve, which is concave to the origin, is represented in Fig. 1 .

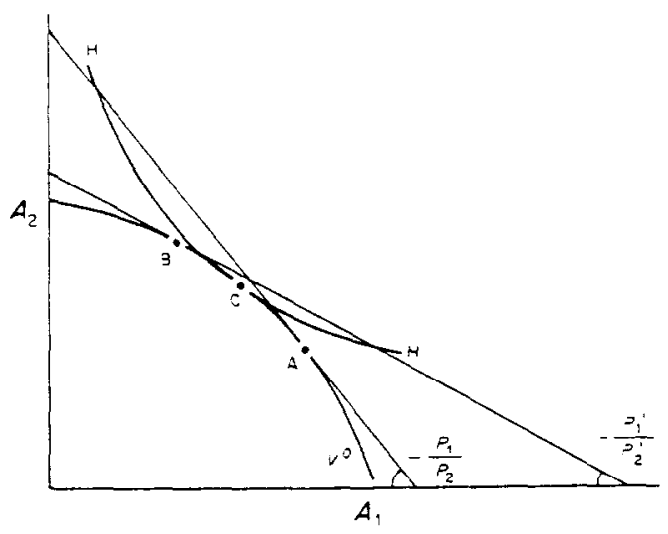

Fig. 1. Production constrained revenue maximization.

This particular shape of the transformation curve is obtained because the production technology exhibits a trade-off: it is impossible to combine both applications in every visit and the use of each of them is limited by the number of visits $V^{0}$. Given this product transformation constraint, the supplier can try to reach the maximal revenue by choosing the combination of $A_{1}$ and $A_{2}$ indicated by the tangency locus of the revenue line and the production frontier. In Fig. 1 this is point $A$, where the relative output price ratio $\left(P_{1} / P_{2}\right)$ equals the marginal rate of transformation between $A_{1}$ and $A_{2}$. It is clear that a change in the relative price ratio from $P_{1} / P_{2}$ to $P_{1}^{\prime} / P_{2}^{\prime}$ would induce a shift from point $\mathrm{A}$ to $\mathrm{B}$ : more $A_{2}$ and less $A_{1}$ will be supplied as a consequence of the fact that $A_{2}$ has become more attractive to the supplier in terms of marginal revenue. From the first order conditions of this maximization problem conditional supply functions in terms of relative prices and input can be derived [6].

Theoretically, it is also possible to define health isoquants in this context: these are curves which represent all combinations of $A_{1}$ and $A_{2}$ with which the same health outcome for the patients can be obtained. In Fig. 1 two such health isoquants are drawn convex to the origin, reflecting the assumption of imperfect substitutability between applications [7]. If the physiotherapist acted as a perfect agent for his patients, he would choose that combination of applications on the production possibility frontier which is tangent to the highest attainable health isoquant. Point $C$ in Fig. 1 represents this sort of welfare

Table 1. Fee changes per type of therapy

\begin{tabular}{|c|c|c|c|}
\hline \multirow[b]{2}{*}{ Therapy* } & \multirow[b]{2}{*}{$\begin{array}{c}\text { Standard } \\
\text { duration } \\
\text { (in minutes) }\end{array}$} & \multicolumn{2}{|c|}{ Fee (in Dfl.) } \\
\hline & & $\begin{array}{l}\text { Before } \\
1 / 10 / 79\end{array}$ & $\begin{array}{l}\text { After } \\
1 / 10 / 79\end{array}$ \\
\hline Massage/exercise $(\mathrm{M} / \mathrm{E})$ & 20 & 13,47 & 17,56 \\
\hline \multicolumn{4}{|l|}{ Low-frequency electro } \\
\hline Application (LFEL) & 15 & 11,78 & 13.17 \\
\hline Ultrasound application (ULTRA) & 10 & 11.78 & 8.78 \\
\hline \multicolumn{4}{|l|}{ High-frequency electro } \\
\hline Application (HFEL) & 6 & 11,78 & 5,72 \\
\hline Thermo application (THERMO) & 10 & 11,78 & 8,78 \\
\hline
\end{tabular}

"Some minor therapies which are used infrequently and the tariffs for delivering care at the home of the patient are not reported here.

Source: Curfs [17, p. 84]. 
optimum as the tangency locus between the transformation curve $V^{\circ}$ and the health isoquant $\mathrm{HH}$. Such behaviour would be characterized by the absence of any output price sensitivity on the part of suppliers: the choice of therapy mix would not be influenced by relative prices.

Much of the health care supply literature of the last decade has concentrated on the question of whether providers (mostly physicians) can and do influence the demand for their services. Evans [8] has pointed out that the crucial empirical issue is not whether providers manipulate their patient's demand to increase their own income, but whether they fully exploit this ability to do so. Clearly, if they do then, at the optimum, demand becomes again exogenous and the income-maximizing case cannot be distinguished from the supplier-inducement case. The critical empirical test therefore, becomes the identification of unexploited potential to influence demand which varies with the extent of economic pressure on the provider [2].

Traditionally, the frequently observed positive correlation-both cross-sectionally and over time-between the number of providers per capita and both price and quantity traded in equilibrium, was quoted as evidence of supplier-induced demand. Several authors [1,9] have argued that such findings might also be consistent with the standard neo-classical model of income maximization when factors such as quality of care, time prices or the existence of excess demand are taken into consideration. In a similar way, the rapid growth in the recent past of physiotherapeutic care utilization parallel with the growth in provider density in The Netherlands is sometimes quoted as evidence of supplier-induced creation of demand [10]. But this phenomenon can also be explained as an effect of catching up with excess demand at the prevailing zero user prices in the public sector.

Instead of looking at the total quantity of demand, we propose a test of supplier-induced transformation of demand [11]. The revenue-maximization model predicts that the composition of utilization will depend on the relative prices of the treatment com- ponents. An exogenous shift in the incomes, such as the one induced by the restrictive volume measure, should not affect this composition when the ability to manipulate demand was already fully exploited before the change [12]. If substitution towards a higher marginal revenue per unit of time is observed after the exogenous shift in income, this implies that income was not maximized before and therefore that the discretionary power to influence demand was not fully realized. Such an observation can only be explained by a model which assumes that providers do not only derive utility from real income but also from some sort of 'ethical behaviour'. Both objectives are traded-off against each other and it is only when income is reduced exogenously, and therefore the marginal utility of income is raised, that providers are willing to suffer the marginal disutility of demand manipulation [1]. In the empirical analysis, we will attempt to test for such behaviour by investigating whether the mix of services provided is affected by the exogenously induced income reduction through the government limitation on the applications per visit ratio.

\section{DATA DESCRIPTION}

In order to test the behavioural hypotheses, quarterly data were gathered on physiotherapeutic care utilization in the 36 practices of the sickness fund region of North Limburg from 1978 to 1981. This sample cannot be said to be representative of the whole country but it is the only sickness fund with these data available over the considered period and there is no reason to expect physiotherapist behaviour in this region to be different from the rest of the country.

Figure 2 shows the aggregated quarterly data for the number of visits (VIS), exercise/massage therapy $(\mathrm{M} / \mathrm{E}$ ) and applications (APPL) over the four year period. At least three observations can be made from this graph. First, there is a consistent seasonal variation in utilization with substantial reductions in the summer quarter (July-August-September). Secondly, gradually the number of massage therapies has ap-

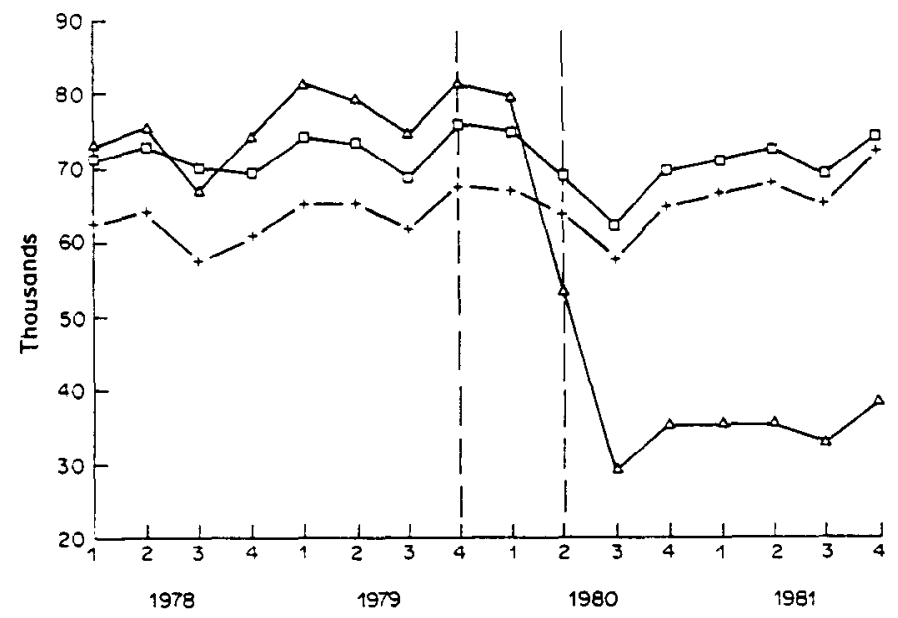

Fig. 2. Physiotherapy utilization rates (quarterly data $1978-1981$ ). ( $\square$ ) Visits; (+) M/E therapy; $(\triangle)$ applications. 
proached the number of visits so that by the end of 1981 almost all consultations include massage therapy. This is not at all surprising since we know that this type of therapy was remunerated higher after the first measure and the volume of other therapies restricted after the second measure. And thirdly, we see a sharp decline in the total number of applications after the implementation of the volume-restricting measure in the second quarter of 1980 . The quantity of applications stabilizes after a few quarters at a level which is about half the level before the intervention.

More interesting than what happened to the total number of applications is the evolution of the relative shares of the specific types of applications within this total. These are depicted in Fig. 3 for the four main categories of applications which together account for about $97 \%$ of the total. The relatively stable pattern of the distribution between the four types of therapy before the interventions has changed drastically at the end of 1979. The share of high-frequency electrotherapy (HFEL) dropped sharply from $45 \%$ to about $30 \%$ whereas the shares of both low-frequency electrotherapy (LFEL) and thermal therapy (THERMO) went up almost simultaneously. Only the share of ultrasound (ULTRA) remained fairly stable and virtually unaffected by the price and voiume regulation. This means, of course, that in absolute quantity terms uitrasound therapy fell at the general rate of decrease in the number of physiotechnic applications. This conforms to the fact that ultrasound is used only for some rather well-determined indications and cannot easily substitute other applications or be substituted for by others.

\section{SPECIFICATION AND ESTIMATION OF STRLCTLRAL EQUATION MODEL}

In order to quantify some of the above mentioned effects and to test for their statistical significance, we specified the following structural equation model:

Equation 1:

$$
\begin{aligned}
\ln \mathrm{M} / \mathrm{E}=\alpha_{0}+ & \beta_{0} \ln \text { VIS } \\
& +\gamma_{0} \mathrm{DPRICE}+\delta_{0} \ln \mathrm{APPL}+\epsilon_{0}
\end{aligned}
$$

Equations 2 to 5 :

$$
\begin{aligned}
\ln \frac{A_{i}}{A}=x_{i}+\beta_{i} & \ln \text { VIS }+\gamma_{i} \text { DPRICE } \\
& +\delta_{i} \ln \text { APPL }+\epsilon_{i} \quad(i=1,2,3,4) .
\end{aligned}
$$

Equation 1 explains the (logarithm of the) number of massage/exercise $(M / E)$ therapies by the number of visits, the dummy variable DPRICE indicating the shift in prices and the total number of applications (APPL). The latter variable should capture the effect of the volume measure. Because of the loglinear specification, the parameters $\beta_{0}$ and $\delta_{0}$ can be interpreted as elasticities and $\%_{0}$ as the percentage rate of change in $\mathrm{M} / \mathrm{E}$ due to the price change. Because all prices changed simultaneously, it is statistically impossible to estimate own and cross price elasticities separately.

The only difference between equation $I$ and equa tions $2-5$ is that not absolute quantities but relative shares of applications are the dependent variables. Consequently, the $\beta_{i}$ 's and the $\delta_{i}$ 's represent share elasticities and the $\gamma_{i}$ 's rates of change in relative shares. The system of equations has to be estimated simultaneously because of the dependency between the disturbance terms which is introduced by the sum constraint [13]

$$
\sum_{i=1}^{4} \frac{A_{i}}{A}=1 .
$$

In that case the contemporaneous correlation between the $\epsilon_{i}$ 's cannot be assumed to be zero. The covariances between the disturbance terms can be estimated either by a generalized least squares (e.g. Zellner's seemingly unrelated regressions estimator) or by a maximum likelihood method. Both estimation procedures have identical asymptotic properties [14]. We have estimated the system by the full information maximum likelihood method of the LISREL $V$ program [15]. The results are presented in Table 2 .

In general, the model fits the data reasonably well: except for the ultrasound equation, the explanatory power indicated by the $\bar{R}^{2}$ is fairly high and the

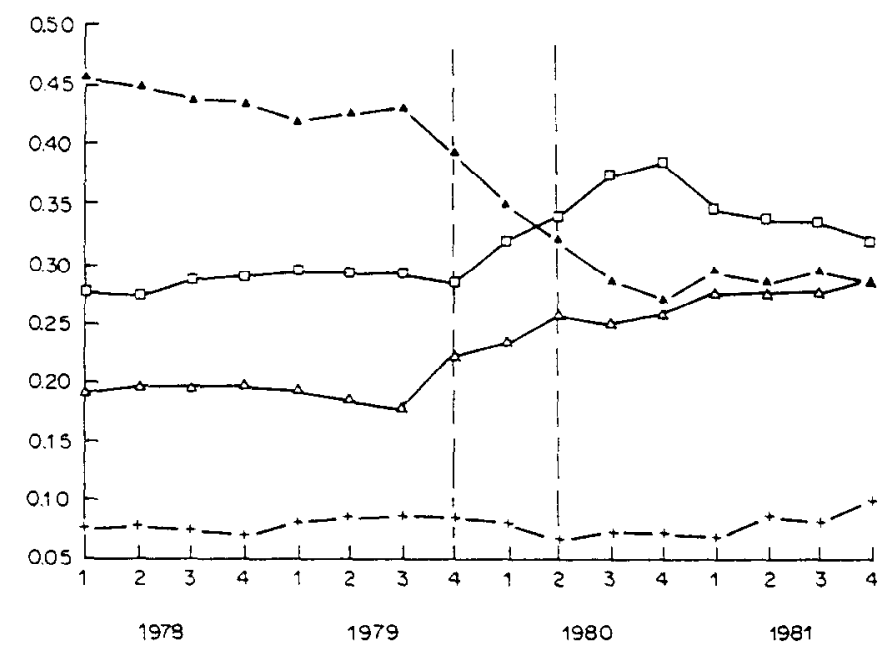

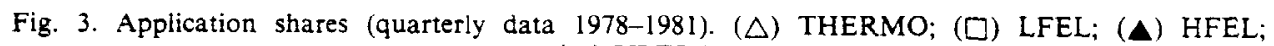
(+) ULTRA. 
Table 2. Full information maximum likelihood regression results

\begin{tabular}{lcccccc}
\hline & CONST & VIS & APPL & DPRICE & $R^{*}$ & D.W. \\
\hline M:E & -1.261 & 1.168 & -0.067 & 0.025 & 0.805 & 2.08 \\
& $(0.68)$ & $(6.35)$ & $(2.17)$ & $(1.25)$ & & \\
LFEL & 6.677 & -0.611 & -0.097 & 0.107 & 0.790 & 1.65 \\
& $(1.74)$ & $(1.61)$ & $(1.53)$ & $(2.56)$ & & \\
HFEL & 0.337 & -0.334 & 0.318 & -0.175 & 0.951 & 2.05 \\
& $(-10)$ & $(1.04)$ & $(5.92)$ & $(4.97)$ & & \\
ULTRA & -17.021 & 1.394 & -0.097 & -0.025 & 0.260 & 1.47 \\
THERMO & $(2.27)$ & $(1.88)$ & $(0.78)$ & $(0.31)$ & & \\
& -7.300 & 0.759 & -0.250 & 0.182 & 0.963 & 1.60 \\
\hline
\end{tabular}

$\bar{R}^{2}$ is the coefficient of determination, adjusted for degrees of freedom. D.W. is the Durbin-Watson test statistic for first order serial correlation. Absolute t-values in parentheses. Only the covariance of the disturbance terms of the LFEL- and HFELequations was significant: $\operatorname{COV}\left(\epsilon_{1}, \epsilon_{2}\right)=-0.001(t$-value $=2.15)$.

Durbin-Watson statistic rejects the test for first-order serial correlation. The interpretation of the first equation is different from the other four equations. The parameter $\beta_{0}$, the visits elasticity, is close to 1 and highly significant, which means that $\mathrm{M} / \mathrm{E}$ therapy follows the general seasonal and trend variation expressed by the number of visits. The applications elasticity $\delta_{0}$ equals -0.06 and is also statistically significant. This indicates that, as a reaction to the governmental restriction of applications by about $50 \%$, physiotherapists have increased the number of $\mathrm{M} / \mathrm{E}$ therapies by about $3 \%$.

Finally, as a result of the price shift, $M / E$ therapy has increased only by about $2 \%$ and this effect is not statistically significant at conventional confidence levels. The finding that both the volume and the price effect on $M / E$ therapy are so small is not surprising because $M / E$ was already applied during more than $90 \%$ of the visits before the changes occurred so that, holding visits constant, this percentage could only slightly be raised.

The coefficients in the application share equations need to be interpreted with caution. If some estimates do not differ significantly from zero this does not mean that these variables do not affect the total supply of this service but simply that the effect does not differ from that on the total amount of applications delivered. Consequently, the relative share remains unaltered. For instance, we notice that the seasonal and trend variation captured by VIS only has a significant positive effect on the share of THERMO.

The question we are mainly interested in is how the mix of application services has changed after the introduction of the government measures. It can be seen from the coefficients of DPRICE that LFEL has gone up by $10.7 \%$, HFEL has decreased by $17.5 \%$, ULTRA was unaffected and THERMO went up by $18.2 \%$ after 1 October, 1979. This implies positive price elasticities for LFEL and HFEL, a zero price elasticity for ULTRA and a negative price elasticity for THERMO [16]. However, this interpretation overlooks some prior information on product transformation possibilities in physiotherapy. Both LFEL and THERMO can in some cases be substituted for HFEL but are rather poor substitutes for each other [17]. This means that we have to look at the relative price changes of these types of therapy compared to HFEL. If we take this into account all price effects are positive, even for THERMO because, relative to HFEL, its price has also risen.

This shift of the treatment mix towards the bigher revenue application was compounded by the second measure which restricted the number of applications that could be done during one visit. This can be seen from the coefficients for APPL which measure the share elasticities with respect to the total number of applications: positive (0.32) for HFEL, negative for THERMO $(-0.25)$ and LFEL $(-0.10)$ and nonsignificant for ULTRA. This implies that, ceteris paribus, the decrease in applications by approx. $50 \%$ has resulted in a $16 \%(=0.32 \times 50)$ drop of the HFEL share which was compensated by a rise in the shares of LFEL and THERMO by respectively 5 and $12.5 \%$. Or, once more, a shift towards the more attractive applications in terms of marginal revenue.

The latter finding points to some degree of unexploited ability to manipulate the mix of services before the volume-restricting measure was introduced. We cannot conclude anything about the appropriateness of the different application mixes because we could not measure any outcomes in terms of patient's health status. However, the fact that no evidence was reported of effects on health outcomes does cast some doubt on the effectiveness of the $100 \%$ higher rates of applications that were performed before the restrictive measure. In general, the multiple regression analysis confirms the picture which already emerged from Figs 1 and 2: both the price and volume measure have had a strong impact on the number of services delivered by physiotherapists. As a reaction to both interventions, physiotherapists altered the average mix of massage/exercise and application therapy towards a higher marginal revenue per unit of time.

\section{CONCLUSION AND POLICY IMPLICATIONS}

The analysis of physiotherapeutic utilization in sections 4 and 5 does lend a considerable amount of support to the hypothesis that changes in relative prices work as strong incentives for suppliers to alter the mix of services supplied. As a reaction to the restriction on the number of applications and to the relative price rise of exercise/massage therapy the utilization of this type of therapy has increased such that it is currently being performed in almost every visit. Secondly, all applications show a significant 
positive own price elasticity except for ultrasound which did not show any price sensitivity. Thermal therapy was the only exception with a negative own price responsiveness but this was shown to be the result of not taking into account that the fee for thermal therapy had in fact been raised (instead of lowered) relative to high frequency electrotherapy, its closest substitute. The relative price elasticity was also positive for this type of physiotherapeutic application.

It is therefore concluded that the perfect agency relationship hypothesis had to be rejected. Positive relative price elasticities are, however, still compatible with both the income-leisure maximization and supplier-inducement hypothesis. In order to discriminate between these two alternative explanations it was argued that evidence is needed on incomplete exploitation of the ability to induce demand. The test proposed in this paper is to see whether the therapy mix is altered towards a higher marginal revenue per unit of time if income is reduced exogenously. It turns out that indeed the shares of higher revenue applications (low frequency electrotherapy and thermotherapy) have risen considerably at the expense of the lower revenue substitute (high frequency electrotherapy) when the maximum allowable applications per visit ratio was halved by the public insurance agency. If pure income maximization were the driving force behind physiotherapist behaviour, why then was this different mix of applications not adopted before the restrictive volume measure?

The final question which remains is whether supplier-induced transformation of demand is necessarily a bad thing. The fact that physiotherapists (or health care providers in general) are sensitive to financial incentives could also be used in a positive way by the regulators. If information were collected on the cost-effectiveness of different therapies this could be used as a basis for the determination of the fee schedule. Our analysis certainly predicts that when cost-effective therapies are rewarded more, they will be substituted for less cost-effective ones. If, on the other hand, such information is unlikely to become available in the near future due to the problems associated with the comparability of physiotherapy evaluation study results [18], a casepayment financing system is worth considering. When physiotherapists are paid a fixed amount of money per patient treated and preferably adjusted for diagnosis, then they can themselves decide on the mix of services to be provided for this amount [19]. Free choice for the patients to choose between providers (competing with quality, not with prices) should guarantee that quality is not lowered due to underprovision of services. The incentive for supplierinduced substitution of demand under such a closedend financing system would then possibly take the form of choosing the cost-minimizing combination of therapies in order to maximize residual revenue. But such behaviour may be preferable to the current situation in the open-ended fee-for-service financing system.

Acknowledgements - The authors would like to thank the Sickfund 'Noord-Limburg' for providing the data, E. Curfs for expert physiotherapeutic advice and F. Tan, G. Haan,
E. Schut and the Maastricht and Rotterdam members of the research project "Health insurance and medical consumption' for their comments on an earlier draft.

\section{REFERENCES}

1. Pauly M. V. Doctors and Their Workshops. Economic Models of Physician Behaviour. N.B.E.R. University of Chicago Press, Ill., 1980.

2. Reinhardt U. E. The theory of physician-induced demand: reflections after a decade. J. Hlth Econ. 4, 187-193, 1985.

3. We will not discuss the physiotherapeutic care provided to private patients ( $30 \%$ of the Dutch population) who are often not completely insured for these expenses.

4. Wennberg J. E. Professional uncerainty and the problem of supplier-induced demand. Soc. Sci. Med. 16, 811-824, 1982.

5. Monsma G. N. Jr. Marginal revenue and the demand for physician's services. In Empirical Studies in Health Economics (Edited by Klarman H.), Johns Hopkins Press, Baltimore, and, 1970 , showed that physicians also alter the mix of services provided in order to increase marginal revenue obtained.

6. Layard P. R. G. and Walters A. A. Micro-Economic Theory, p. 219. McGraw-Hill, New York, 1978.

7. The general assumption of a direct elasticity of substitution between zero and infinity seems reasonable. It implies that the relative marginal health product of an application diminishes when its use intensity is increased relative to the other, holding health constant.

8. Evans R. G. Supplier-induced demand: some empirical evidence and implications. In The Economics of Health and Medical Care (Edited by Perlman M.). Wiley, New York, 1974

9. Reinhardt U. E. Comment on Sloan F. A. and Feldman R. Competition among physicians. In Competition in the Health Care Sector: Past. Present and Future (Edited by Greenberg W.), pp. 45-102. Aspen Systems, Germantown, 1978.

10. Van de Ven W. P. M. M. Ziektekostenverzekering en de collectieve sector (Health insurance and the public sector). In De Collectieve Sector in de Crisis (Edited by Brouwer M. T. and Ellman M. J.). pp. 267-297. Kluwer, Deventer, 1983

11. The distinction between demand and supply is often difficult to establish in health care and it may therefore be better to speak of utilization.

12. This reasoning implicitly assumes that the limitation on the number of applications per visit induces a parallel inward shift of the production possibility frontier. The marginal rate of transformation between all outputs (applications) remains the same and, since the price ratio is unchanged, the optimal application shares should be unaffected if revenue maximization is pursued.

13. Imposing a structure on the model which would make it inherently satisfy this sum constraint (e.g. the class of attraction models) would be preferable but impossible to estimate in our case because the explanatory variables are the same in each equation. See Bultez A. V. and Naert $\mathrm{Ph}$. A. Consistent sum-contrained models. J. Am. stat. Ass. 70, 529-535, 1975 .

14. Kmenta J. Elements of Econometrics, p. 526. Macmillan, New York, 1971.

15. Jöreskog K. G. and Sörbom D. LISREL: Analysis of Linear Structural Relationships by the Method of Maximum Likelihood. User's Guide, Version V. International Educational Services, Chicago, Ill., 1981.

16. The elasticities can be calculated as follows: the relative price changes, defined as $\left(P_{2}-P_{1}\right) / P_{1}$, with $P_{1}$ equal to the price before and $P_{2}$ after the fee measure, can be found in Table 1. They are for respectively LFEL, 
HFEL and THERMO equal to $12,-51$ and $-25 \%$. The implied share elasticity figures are therefore: 0.89 , 0.34 and -0.73 .

17. We thank E. Curfs for making this point in Curfs E. C. Effecten van Overheidsmaatregelen ten aanzien can de Fysiotherapie (Effects of Government Regulation of Physiotherapy). Rijksuniversiteit Limburg, Maastricht, 1985.
18. Partridge C. J. The effectiveness of physiotherapy. Physiotherapy 66, 153-155, 1980.

19. For a successful experiment with such a capitation payment for drug expenses of Medicaid recipients in the U.S. and the impact on generic substitution. See Yesalis C. E. et al. Capitation payment for pharmacy services; impact on generic substitution. Med. Care 18, 816-828, 1980. 